\title{
Caso clínico: aproximación a la vía aérea difícil en neonatología. Síndrome de Treacher-Collins
} Clinical case: approach to the difficult airway in neonatology. Treacher-Collins
syndrome

\author{
Dr. José P. Marmo Lupano ${ }^{a}$ Dra. María S. Medina ${ }^{a}$, Dr. Juan J. Bosch ${ }^{b}$, Dra. Leticia Negrinc, \\ Dr. Guillermo Arteroc, Dr. Ernesto Goldschmidt ', Dra. Carolina Josée, Dra. Sandra B. Díaz \\ Dr. Agustín Bernatzky y Dr. Juan P. Berazategui ${ }^{a}$
}

\begin{abstract}
RESUMEN
Cuando los recién nacidos presentan obstrucción dela vía aérea, requieren un manejourgente y experto para evitar la mortalidad y la morbilidad. La definición de vía aérea difícil se relaciona con problemas en la intubación endotraqueal o en la ventilación a presión positiva con bolsa y máscara o reanimador de pieza en T. El manejo debe basarse en la comprensión del mecanismo fisiopatológico responsable de la vía aérea difícil. Las causas en el recién nacido pueden ser congénitas y/o adquiridas.

Se presenta el caso de una recién nacida con síndrome de Treacher-Collins tipo 1 [OMIM \#154500] con una disostosis mandibulofacial, micrognatia, hipoplasia malar, paladar hendido, sin cardiopatía congénita, asociado con intubación extremadamente difícil.

Palabras clave: obstrucción de las vías aéreas, neonatología, síndrome de dificultad respiratoria, reanimación cardiopulmonar, síndrome de Treacher-Collins.
\end{abstract}

\begin{abstract}
If newborns have an airway obstruction, they require urgent and expert management to avoid mortality and morbidity. The definition of difficult airway includes problems in endotracheal intubation or positive pressure ventilation with bag and mask or T-piece resuscitator. Management should be based on an understanding of the pathophysiological mechanism responsible for difficult airway. The causes of difficult airway in the newborn can be congenital or acquired.

We present the case of a newborn with Treacher-Collins syndrome Type 1 [OMIM \# 154500] with a mandibulofacial dysostosis, micrognathia, malar hypoplasia, cleft palate, without congenital heart disease, associated with extremely difficult intubation.
\end{abstract}

a. Unidad de Neonatología.

b. Endoscopía Respiratoria y Neumología Infantil.

c. Cirugía Plástica y Maxilofacial.

d. Genética.

e. Oftalmología.

Sanatorio Anchorena San Martín, Buenos Aires, Argentina.

Correspondencia:

Dr. Juan P. Berazategui: jberazategui@sasm.com.ar

Financiamiento: Ninguno.

Conflicto de intereses: Ninguno que declarar.

Recibido: 9-6-2020

Aceptado: 25-8-2020
Key words: airway obstruction, neonatology, respiratory distress syndrome, cardiopulmonary resuscitation, Treacher-Collins syndrome.

http: / / dx.doi.org/10.5546/ aap.2021.e45

Cómo citar: Marmo Lupano JP, Medina MS, Bosch JJ, Negrin L, et al. Caso clínico: aproximación a la vía aérea difícil en neonatología. Síndrome de Treacher-Collins. Arch Argent Pediatr 2021;119(1):e45-e48.

\section{INTRODUCCIÓN}

La intubación endotraqueal (IET) de los recién nacidos (RN) es un procedimiento que requiere entrenamiento y destreza. En nuestro país, es competencia exclusiva del médico. Por lo tanto, es necesario adquirir el entrenamiento en el manejo de la vía aérea difícil (VAD), ya que, en neonatología, es una emergencia por resolver.

Las características del síndrome de TreacherCollins (STC) tipo 1 son un ejemplo de este escenario. Engloba la presencia de micrognatia, deficiencia malar de tejidos blandos y duros, paladar hendido, hendiduras palpebrales con desviación ínfero-externa, euriblefarón con o sin coloboma y pérdida auditiva conductiva. ${ }^{1,2}$

En estos RN, la osteogénesis por distracción mandibular (ODM) es una de las principales herramientas reconstructivas en la actualidad, ${ }^{1,2}$ que ha demostrado su efectividad para promover el crecimiento y la expansión ósea de la matriz de tejido blando perimandibular, particularmente, en los pacientes pediátricos. ${ }^{3,4}$ Se presenta a una paciente RN con VAD secundaria a una malformación craneofacial congénita.

\section{CASO CLÍNICO}

Embarazo controlado, segunda gesta, sin antecedentes patológicos. Cesárea programada por otra previa. RN de sexo femenino, a las 39 semanas de edad gestacional, de peso adecuado, con presentación podálica. Se detectó polihidramnios. Se observó facies peculiar, dificultad para iniciar 
respiraciones en forma espontánea y bradicardia. Se decidió el clampeo precoz del cordón umbilical; se trasladó a la sala de recepción y se inició la ventilación a presión positiva (VPP) con reanimador de pieza en $\mathrm{T}$.

$\mathrm{Al}$ momento del examen físico inmediato, se describió microrretrognatia, glosoptosis y paladar hendido, hendiduras palpebrales con desviación ínfero-externa, microtia bilateral. Presentó buena respuesta a VPP con tracción anterior de la mandíbula. Se solicitó a un segundo neonatólogo

FIGURA 1. Estabilización de la vía aérea con máscara laríngea

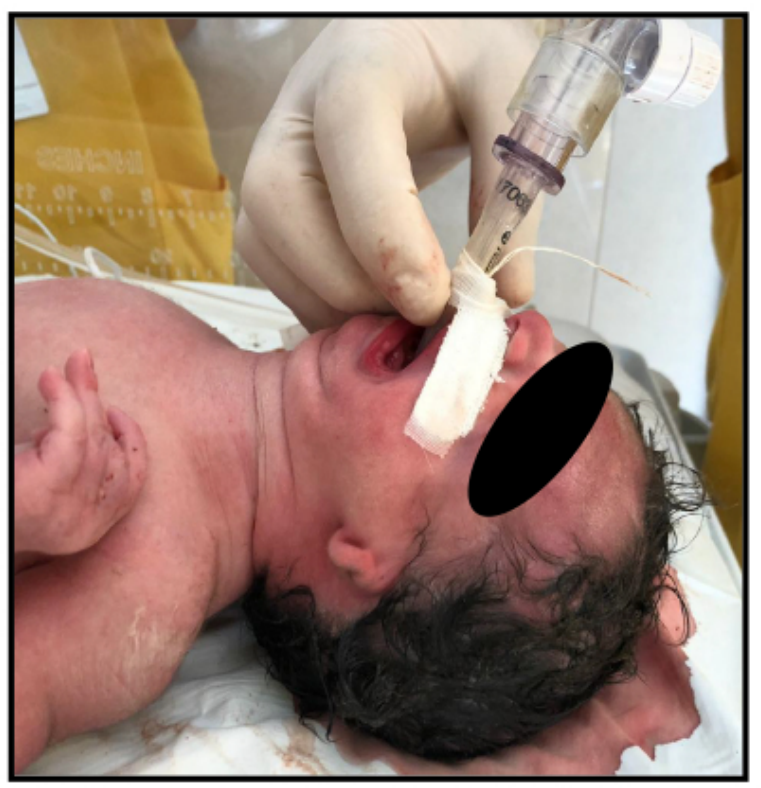

y se decidió la IET, en la que se observó VAD. No se logró la intubación en el primer intento, por lo que se requirió la asistencia de un neonatólogo con mayor experiencia quien logró estabilizar la vía aérea con máscara laríngea (ML), lo que permitió la ventilación en forma efectiva y espontánea (Figura 1). Durante el proceso, se convocó a un endoscopista respiratorio.

Ya estable, con fracción inspirada de oxígeno $\left(\mathrm{FIO}_{2}\right)$ del $21 \%$, la paciente se trasladó a la Unidad de Cuidados Intensivos. Se realizó la canalización umbilical y, posteriormente, ingresó a quirófano, donde se sometió a broncoscopía y traqueotomía (Figura 2). Con evolución respiratoria favorable, se realizó el destete de asistencia respiratoria mecánica a las 36 horas de vida.

La paciente fue abordada en forma multidisciplinaria. Se evaluó por Genética, cuya impresión diagnóstica clínica fue de STC tipo 1, con cariotipo femenino normal (46 XX). Cirugía Plástica decidió, luego de su evaluación clínica y de una resonancia magnética nuclear (RNM) de cráneo y macizo facial, programar la cirugía de ODM al $9^{\circ}$ día de vida.

La paciente egresó del Sanatorio con internación domiciliaria, sin requerimiento de oxígeno, alimentándose por sonda. Continúa con distractores mandibulares en fase de consolidación, en la $4^{\text {a }}$ semana de 6 totales (cumplió la fase de activación y distracción diaria de 3 semanas). Se halla en seguimiento semanal de Cirugía Plástica, Oftalmología, Otorrinolaringología y Fonoaudiología terminada la ODM (Figura 3).

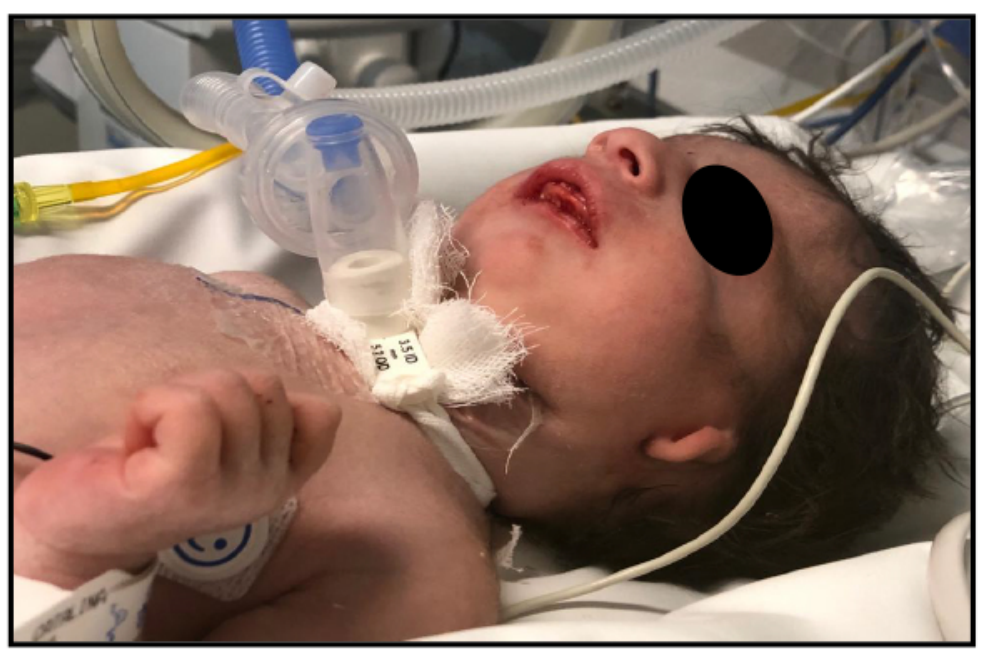




\section{DISCUSIÓN}

El STC tipo 1 es una entidad nosológica de etiología monogénica debida a diversas mutaciones del gen TCOF1 ubicado en el brazo largo del cromosoma 5 (5q32-q33.12) con un patrón de herencia autosómica dominante. El tipo 2 y el tipo 3 se deben a mutaciones de los genes POLR1C y POLR1D, con herencia autosómica dominante para el tipo 2 y recesivo para el tipo 3. Los signos fenotípicos más frecuentes son hendiduras palpebrales con desviación ínfero-externa, coloboma de párpados, micrognatia, microtia y otras dismorfias auriculares, hipoplasia de los arcos zigomáticos y macrostomía. Es frecuente que los afectados presenten hipoacusia conductiva y paladar hendido. La incidencia es de alrededor de 1 en 50 000, sin predilección sexual. La evolución neurológica y madurativa suele ser normal. ${ }^{5}$

El caso presentado, a pesar de poseer controles adecuados, no permitió realizar el diagnóstico prenatal, por lo que se transformó en una recepción habitual en una emergencia neonatal. La provisión rápida de ventilación efectiva es el mejor predictor individual de reanimación neonatal exitosa. ${ }^{6} \mathrm{Al}$ nacer, solo el $5 \%$ de los recién nacidos a término (RNT) requieren mayor asistencia que el secado y la estimulación; el $3 \%$ responderá con VPP y solo un $2 \%$ requerirá IET como sostén ventilatorio. ${ }^{7}$ Hay situaciones en las que la IET puede ser difícil, por ejemplo, en neonatos muy prematuros y en los que presentan anomalías craneofaciales (como nuestra paciente).
Cuando se anticipa el escenario, la recepción es realizada por el especialista en Neonatología. Ante la falta de predictores que anticipen una recepción dificultosa, esta suele ser llevada a cabo por residentes de Neonatología y/ o Pediatría, pediatras o generalistas entrenados en recepción de RN no patológicos. Se sabe que la habilidad en la IET de los RNT, prematuros extremos y con malformación congénita es un requisito para completar la especialidad de Neonatología. Sin embargo, esta competencia en el manejo de las vías aéreas tiende a ser oportunista y, a menudo, la experiencia individual es variable. Se considera que, antes de cualquier recepción, es importante evaluar lo siguiente:

1. Si hay predictores de VAD: considere reunir a un equipo completo de reanimación antes del nacimiento, junto con los recursos necesarios. Es fundamental el nivel de entrenamiento y capacitación de la persona que lidera la recepción porque será importante en la conducción. ${ }^{8}$

2. Si las dificultades con la ventilación y/o IET ocurren de manera inesperada (como en el caso de esta paciente), independientemente de la experiencia y/o capacitación, siempre se debe solicitar ayuda en forma precoz, junto con el equipamiento necesario para el manejo de la VAD, intentando mantener una ventilación efectiva.

3. Si la ventilación y/o intubación no es posible, se debe lograr asegurar una adecuada $\mathrm{VPP}$, con reanimador con pieza en $\mathrm{T}$ o $\mathrm{BM}$,

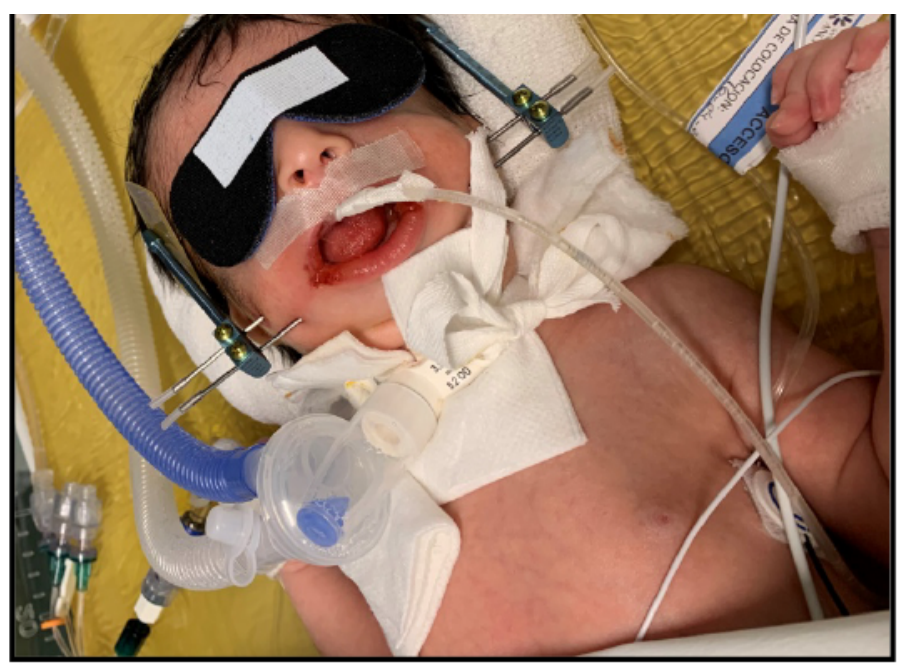


reposicionar al paciente y corroborar el sellado de la máscara facial. De ser necesario, se debe realizar el sellado con 2 manos y tracción mandibular (requiere 2 operadores) y evaluar la necesidad de la ML. La intubación debe ser realizada por el operador más experimentado y nunca superar los 2 intentos por operador, con un máximo de 4 intentos totales. De esta manera, se evitará transformar una vía aérea que "puedo ventilar, no puedo intubar" en "no puedo intubar, no puedo ventilar". Lo más importante es mantener una ventilación adecuada.

En nuestro caso, se utiliza la opción de la ML como una forma de estabilización de la vía aérea y es en este punto donde se desea poner énfasis: la utilización de ML ante un RN con anomalías craneofaciales debería ser la primera opción para estabilizar la vía aérea en lugar del IET. Su colocación es simple, permite asegurar la vía aérea y esperar a un especialista. ${ }^{9}$ Se cree que su falta de uso está relacionada con no tener este recurso disponible, pero también en el común de pensar que la IET es superior a la ML en la estabilización de la vía aérea a pesar de la recomendación de la Sociedad Argentina de Pediatría y de la Academia Americana de Pediatría en el manejo de la VAD.

4. Ante la sospecha o el reconocimiento de una VAD, es importante poder contar con un endoscopista respiratorio presente en el nacimiento o de rápido acceso de ser requerida una IET con fibra óptica o traqueotomía de urgencia. 8,10

Respecto al ODM realizado a los 9 días de vida, no está exento de complicaciones, pero tiene justificación. Una revisión sistemática realizada por Verlinden et al., resume la incidencia de diversas complicaciones, que incluyen infección (el 6,0 \%), parálisis del nervio facial (el 1,8\%), lesión del nervio alveolar inferior (el 1,9\%) y problemas relacionados con el dispositivo (el 7,3\%). ${ }^{11}$

Una de las complicaciones más graves es la anquilosis y la degeneración ósea de la articulación temporomandibular (ATM). Se ha encontrado que la tasa de anquilosis de la ATM varía de manera predecible con diversos factores, incluido el síndrome causante (STC, Goldenhar, Nager, Stickler, etc.), el uso de dispositivos de descarga condilar y la edad. Aunque la correlación exacta entre la patología posoperatoria de la ATM y la edad del paciente sigue siendo debatida, alguna evidencia sugiere que el riesgo de anquilosis de la ATM y otras complicaciones aumenta con la edad. ${ }^{12-14}$

La característica anatómica única de la vía respiratoria superior de los neonatos, a veces, dificulta la laringoscopía y la intubación. La presencia de anormalidades se suma a esta dificultad. En todos los casos de VAD, es obligatorio preservar la respiración espontánea mientras se asegura la vía aérea. La intubación con fibra óptica es la opción ideal, pero el problema, en nuestro país, es su disponibilidad limitada. La ML es la alternativa por excelencia en RN que presentan dificultad para la ventilación, principalmente, en los que se observan malformaciones craneofaciales.

\section{REFERENCIAS}

1. McCarthy JG. The role of distraction osteogenesis in the reconstruction of the mandible in unilateral craniofacial microsomia. Clin Plast Surg. 1994; 2(4):625-31.

2. Scolozzi P, Herzog G, Jaques B. Simultaneous maxillomandibular distraction osteogenesis in hemifacial microsomia: a new technique using two distractors. Plast Reconstr Surg. 2006; 117(5):1530-41.

3. Al-Mahdi AH, Al-Hasnawi SN, Al-Jumaily HA. Changes in soft tissue measurements after mandibular distraction osteogenesis. J Craniofac Surg. 2016; 27(7):e702-7.

4. Monasterio FO, Molina F, Andrade L, Rodríguez C, et al. Simultaneous mandibular and maxillary distraction in hemifacial microsomia in adults: avoiding occlusal disasters. Plast Reconstr Surg. 1997; 100(4):852-61.

5. Caluseriu O, Lowry BR, McLeod R, Lamont $\mathrm{R}$, et al. The Hutterite variant of Treacher Collins syndrome: a 28-yearold story solved. Am J Med Genet A. 2013; 161A(11):2855-9.

6. Johansen LC, Mupanemunda RH, Danha RF. Managing the newborn infant with a difficult airway Infant. 2012; 8(4):116-9.

7. Comité de Estudios Feto-Neonatales (CEFEN). Manual de Reanimación Cardiopulmonar Neonatal. $2^{a}$ ed. Buenos Aires: Sociedad Argentina de Pediatría; 2016.

8. Trevisanuto D, Micaglio M, Pitton M, et al. Laryngeal mask airway: is the management of neonates requiring positive pressure ventilation at birth changing? Resuscitation. 2004; 62(2):151-7.

9. Berazategui JP, Aguilar A, Escobedo M, Dannaway D, et al. Riskfactors for advanced resuscitation in term and near-term infants: a case-control study. Arch Dis Child Fetal Neonatal Ed. 2017; 102(1):F44-50.

10. O'Shea J, Thio M, Kamlin CO, McGrory L, et al. Videolaryngoscopy to Teach Neonatal Intubation: A Randomized Trial. Pediatrics. 2015; 136(5):912-9.

11. Verlinden CR, Van de Vijfeijken SE, Jansma EP, Becking AG, et al. Complications of mandibular distraction osteogenesis for congenital deformities: a systematic review of the literature and proposal of a new classification for complications. Int J Oral Maxillofac Surg. 2015; 44(1):37-43.

12. Sesenna E, Magri AS, Magnani C, et al. Mandibular distraction in neonates: indications, technique, results. Ital J Pediatr. 2012; 38:7.

13. Gerbino G, Bianchi FA, Verzé L, Ramieri G. Unilateral mandibular hypoplasia in adult patients: distraction osteogenesis and conventional osteotomies in a standardized sequence. J Craniofac Surg. 2014; 25(6):1959-66.

14. Kelley P,Mata C,DaSilveira A.Chronictemporomandibular joint dislocation by mandibular distraction in a patient with Melnick-Needles syndrome. J Craniofac Surg. 2010; 21(1):174-6. 